\title{
Relationship between oxygen saturation percentage and forced expiratory volume in first second in chronic obstructive pulmonary disease
}

\author{
$\underline{\text { Seyyed Gholamreza Mortazavi Moghaddam }}^{1}$ (iD), Hosein Gazi ${ }^{2}$ (iD \\ ${ }^{1}$ Corresponding author; Internal Medicine Department, Vali-e-asre Hospital, Birjand University of Medical sciences, Birjand, Iran. \\ Tel:05632435633 Email: gmortazavi@yahoo.com \\ ${ }^{2}$ Internal Medicine Department, Vali-e-asre Hospital, Birjand University of Medical sciences, Birjand, Iran
}

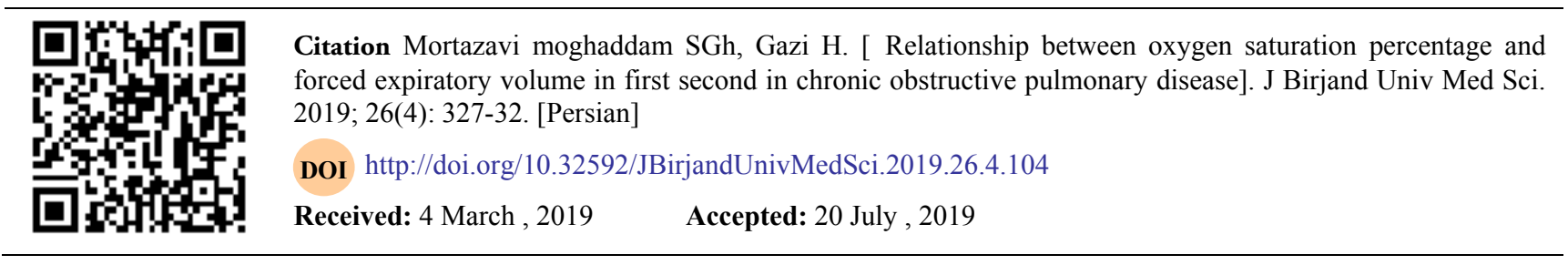

\begin{abstract}
Background and Aim: Considering the cost and some limitations in measuring the percentage of expiratory volume in the first second (FEV1\%), This study was performed to determine the relationship between oxygen saturation percentage (SPO2\%) with expiratory volume in the first-second (\% FEV1) in patients with chronic obstructive pulmonary disease(COPD).
\end{abstract}

Materials and Methods: In a descriptive-analytical study, 25 women and 25 men with COPD were selected by Non-probability and simple sampling from patients referred to the outpatient clinic of Vali-e-Asr Hospital regardless of the severity of the disease. To measure of \% FEV1, spirometry method and a finger pulse oximeter to measure SPO2 was used.Statistical tests including Pearson test and independent t-test and linear regression model were used.

Results: The mean patient age $63.18 \pm 10.47$ years, average \% FEV1 of $36.60 \pm 16.15$ and the mean SPO2\% $84.20 \pm 5.35$ was respectively. The correlation coefficient between $\% \mathrm{FEV} 1$ and SPO2\% was $0.36(\mathrm{P}=0.01)$. Mean of SPO2\% in smokers and nonsmoker was $\% 82.67 \pm 5.22$ and $\% 86.50 \pm 5.29$ respectively $(\mathrm{p}=0.02)$. Regression analysis in constant conditions showed $\% \mathrm{FEV} 1 \mathrm{By}$ increasing one unit $\mathrm{SPO} 2 \%$, the amount of $\mathrm{FEV} 1 \%$ with probability factor of 0.16 will be increased $0.36(\mathrm{P}=0.02)$.

Conclusion: The amount of SPO2\% significant correlation at the medium level with the amount of \%FEV1 and can predict to some extent FEV1\%; Therefore, it has been diagnosed in people with obstructive pulmonary disease and it can be used for evaluation and follow-up of patients instead of spirometry.

Key Words:Chronic Obstructive Pulmonary Disease, Forced Expiratory Volume In First Second, Oxygen Saturation. 


\section{رابطه درصد اشباع اكسيزن با درصد حجم بازدمى ثانيه اول در بيمارى انسدادى مزمن ريه}

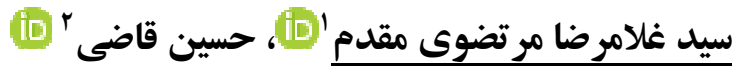

\section{جكيده}

زمينه و هدف: با توجه به هزينه و بعضى محدوديتها در اندازميّى درصد حجم بازدمى ثانيه اول (FEV1\%) (FEV)، اين مطالها

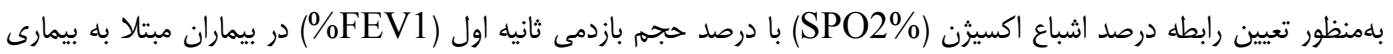
انسدادى ريه (COPD) انجام شد.

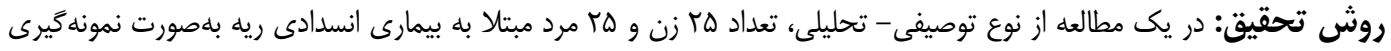

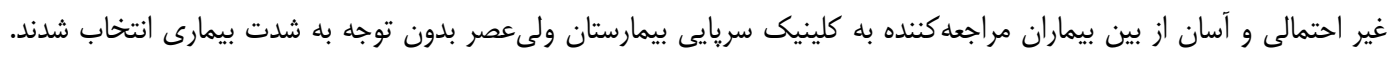

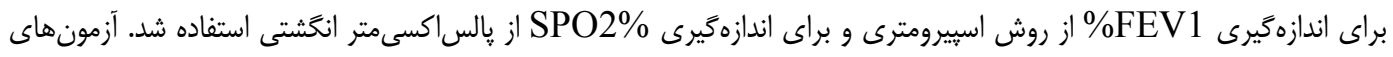

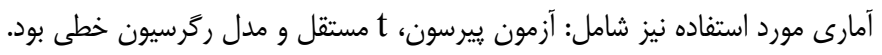

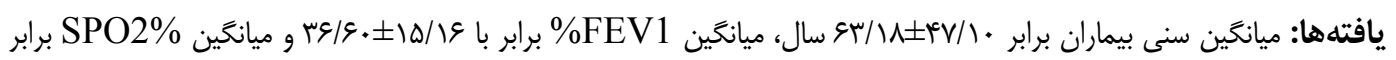

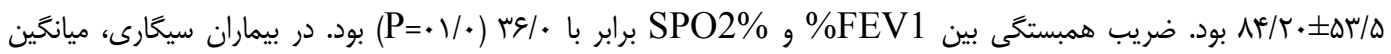

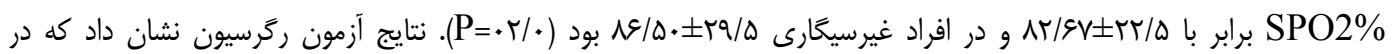

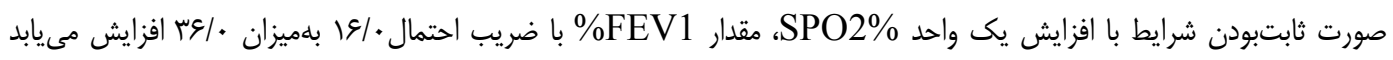
(P=.r/•)

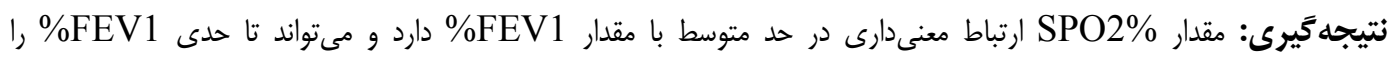

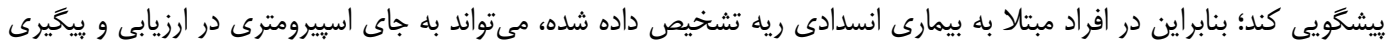
بيماران مورد استفاده قرار كيرد. وازههاى كليدى: بيمارى انسدادى مزمن ريه، جريان حد اكثر بازدمى ثانيه اول، اشباع اكسيثنى

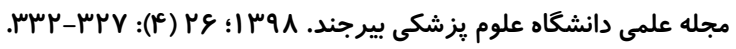
دريافت: س//R/IR 


\section{روش تحقيق}

در يك مطالعه مقطعى از نوع توصيفى - تحليلى در سال

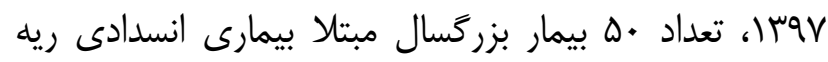

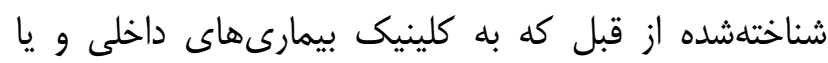
تنفسى بيمارستان ولى عصر بيرجند براى بييخيرىهاى رئى روتين

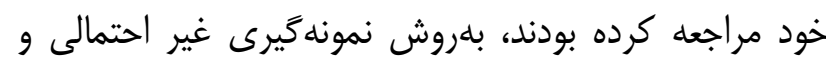

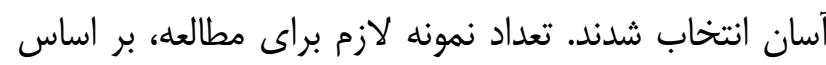

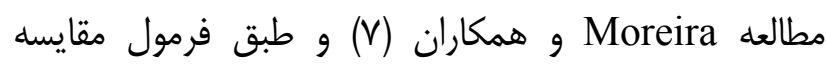

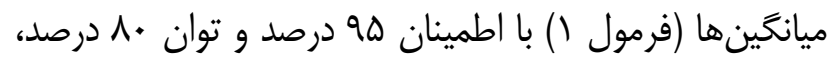
هأ نفر محاسبه شد كه در نهايت با درنظر كرفتن احتمال

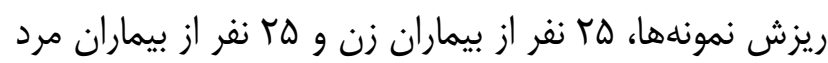
با كسب رضايت كتبى وارد مطالعه شدند.

$$
\begin{aligned}
& \text { فرمول (1): - (1) - (1) }
\end{aligned}
$$

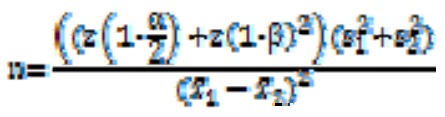$$
z\left(1-\frac{\pi}{2}\right)=1.96
$$$$
z(1-\beta)=0.84
$$

تشخيص COPD بر اساس وجود عوامل خطر COPD، علائم بالينى و تأييد وجود انسداد غير قابل برگشت دراس درد

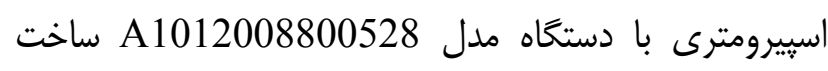

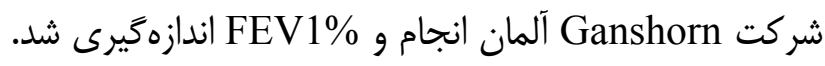
در صورت وجود بيمارىهاى همراه مثل: بيمارىهاى قلبى -

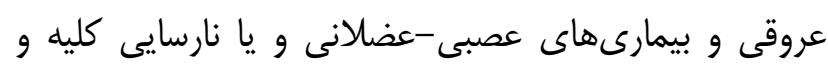

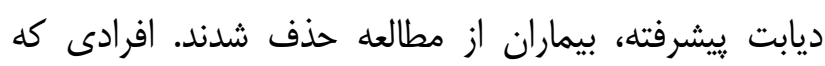

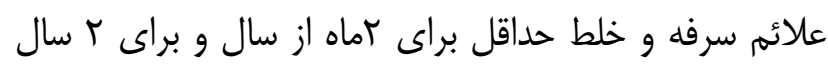

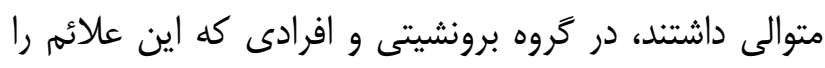

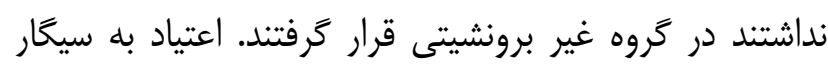

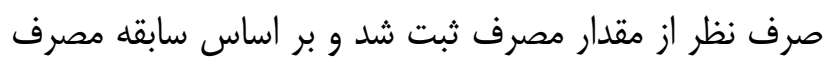

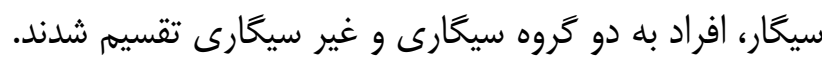

اندازهخيرى درصد حجم بازدمى ثانيه اول (FEV1\%)' به

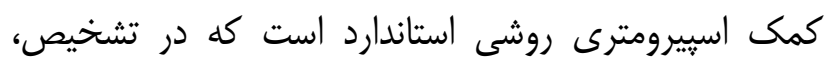

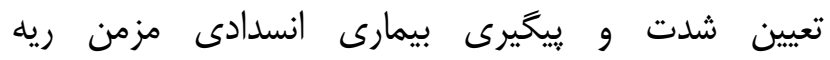
(COPD)

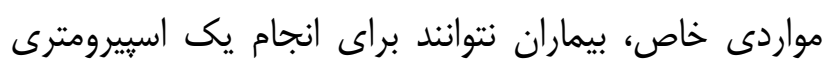

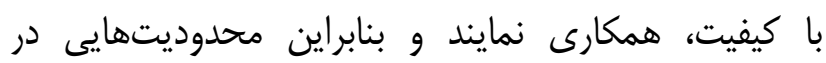

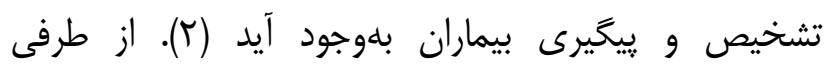

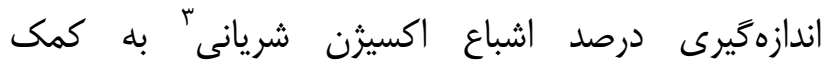

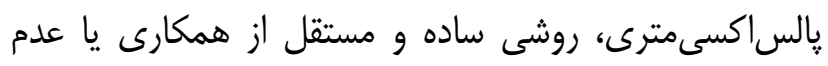

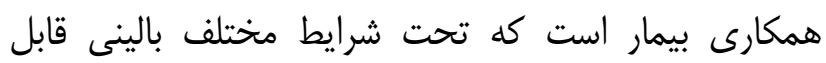

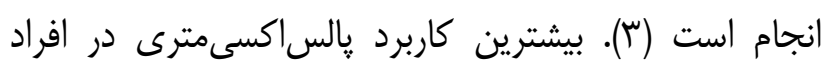

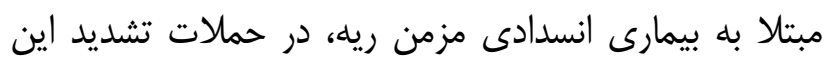

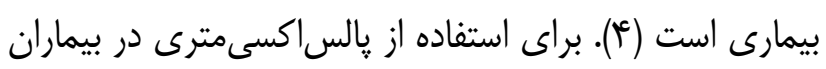
COPD در مواردى غير از حملات (تشديد بيمارى ) COPD نياز است كه مطالعاتى انجام شود. در يك مطالعه كه بلهمنظور إنها

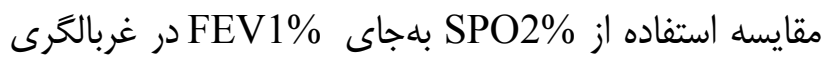
و تشخيص اوليه بيماران COPD انجام شد، جايگزين كردن SPO2\% بهجاى FEV1\% مناسب تشخيص داده نشد (ه)؛

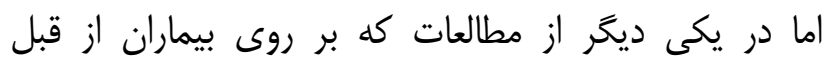
تشخيص داده شده COPD انجام شد، نتايج نشان داد در

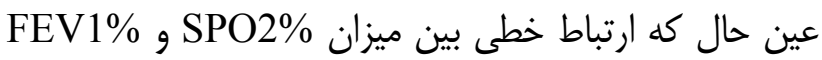
وجود نداشت، ولى بلطور كامل اين فرضيه رد نشد و بر بر انجام مطالعات بيشتر در اين زمينه تأكيد گرديد (و).

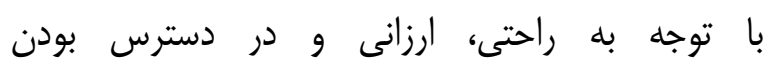

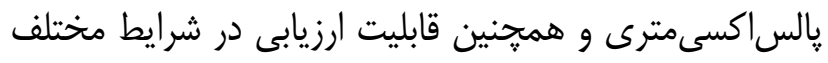

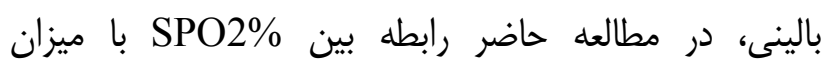

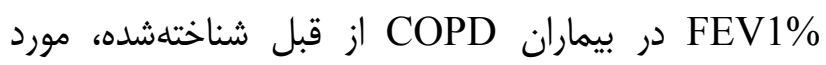

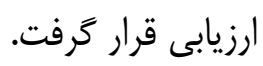

\footnotetext{
${ }^{1}$ Forced Expiratory Volume in one second (FEV1\%)

${ }^{2}$ Chronic Obstructive Pulmonary Disease (COPD)

${ }^{3}$ Pulse Oxygen saturation (\%SPO2)

${ }^{4}$ COPD exacerbation
} 
نمايه توده بدنى (BMI) توسط محقق محاسبه شد و احساس يافتهها

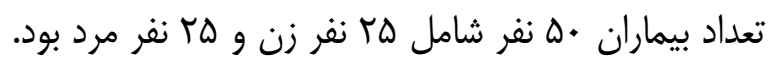

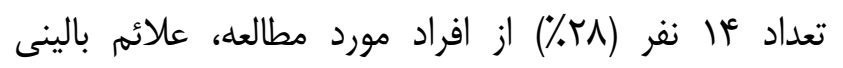

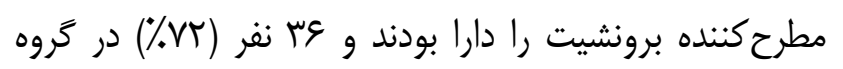
غير برونشيتى قرار كرفتند.

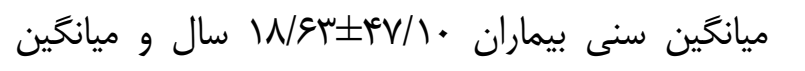
FEV1\% צ/ خصوصيات بيماران در كروهبندىهاى انجامشده (كروه

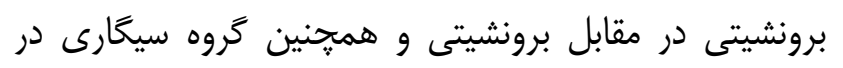
مقابل غير سيخارى) در جدول يك آورده شده است.

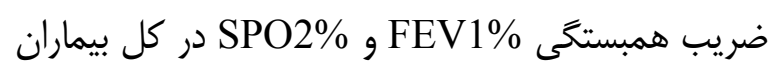

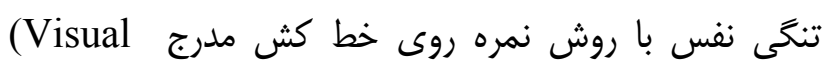
analogue scale, VAS)

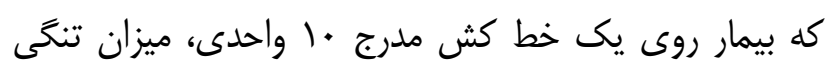
نفس خود را از يك تا •ا (ا كمترين و • •ا بيشترين ميزان تنگى نفس) نمرهذارى كرد. با استفاده از زيالساكسىمتر

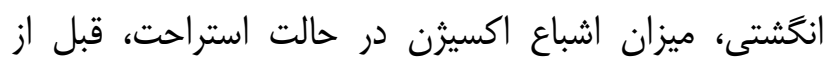

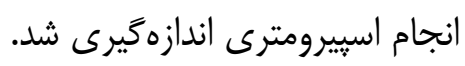

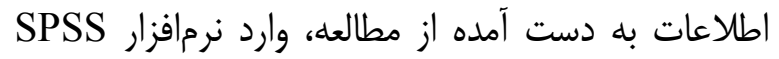

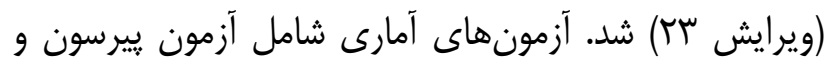

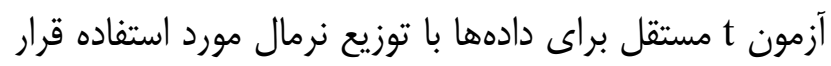

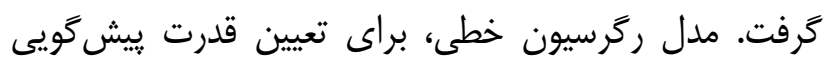

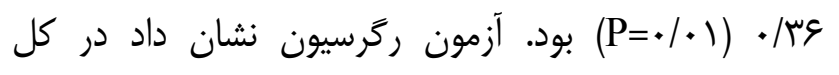

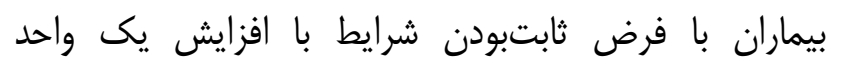

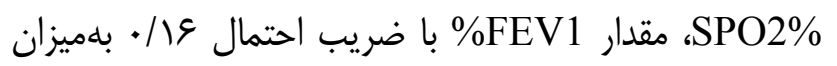

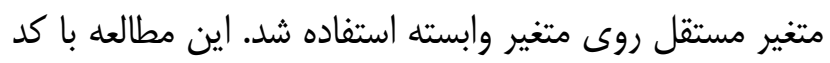

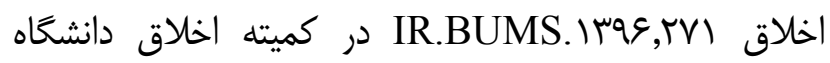

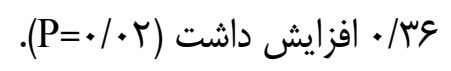
علوم يزشكى بيرجند ثبت شده است.

جدول I - مقايسه يار امترهاى تنفسى، بدنى و سن بيماران در تروههاى مورد مطالعه

\begin{tabular}{|c|c|c|c|}
\hline سيكًار & \multicolumn{2}{|c|}{ علائم برونشيتى } & \multirow[b]{2}{*}{ متغير مورد مطالعه } \\
\hline 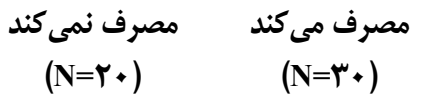 & 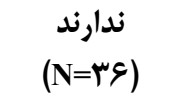 & $\begin{array}{c}\text { دارند) } \\
(\mathrm{N}=\mid f)\end{array}$ & \\
\hline$(\mathrm{T}=r / r \cdot{ } \mathrm{P}=\cdot / \cdot r)$ & \multicolumn{2}{|c|}{$(\mathrm{T}=\cdot / \wedge \cdot ، \mathrm{P}=\cdot / \kappa \mathrm{T} T)$} & درصد اشباع اكسيثنى همو كلوبين \\
\hline$(\mathrm{T}=\cdot / \mathrm{Vr} \cdot \mathrm{P}=\cdot / \notin \varepsilon)$ & \multicolumn{2}{|c|}{$(\mathrm{T}=. / 9 \Delta, \mathrm{P}=. / \Delta))$} & شدت تنكى نفس روى خط كش \\
\hline$(\mathrm{T}=\cdot / \mu \Delta, \mathrm{P}=\cdot / / r)$ & \multicolumn{2}{|c|}{$(\mathrm{T}=\cdot / \& r ، \mathrm{P}=\cdot / \Delta \mathrm{T})$} & 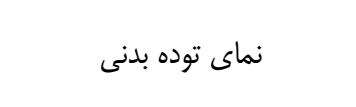 \\
\hline $\begin{array}{c}q 1 / r r \pm r \cdot / r \varepsilon \quad q / / \mathrm{q} \pm \lambda \cdot / r \varepsilon \\
(\mathrm{T}=\cdot / 1 \cdot(\mathrm{P}=\cdot / q 1)\end{array}$ & $\begin{array}{r}\vee q / 1 \Delta \pm \cdot \varepsilon / r q \\
(T=\cdot /\end{array}$ & $\begin{array}{l}\varepsilon \cdot / I V \pm \cdots / / \Lambda \\
P=\cdot / \vee \cdot)\end{array}$ & درصد حجم بازدمى ثانيه اول \\
\hline $\begin{array}{c}(\mathrm{T}=1 / \Delta V \cdot \mathrm{P}=\cdot / \mathrm{T}) \\
9 q / \cdot \pm 1 \cdot / 4 \mathrm{~T} \quad \pm 1 \cdot / 4)\end{array}$ & $\begin{array}{r}G T / \mu q \pm q / T F \\
(T=\cdot /\end{array}$ & 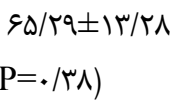 & سن بيماران \\
\hline
\end{tabular}


و آنها نتيجه كرفتند كه اشباع اكسيثنى، مستقل از FEV1\%

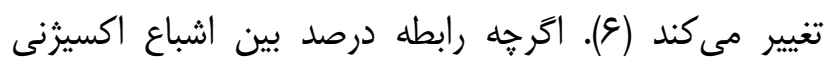

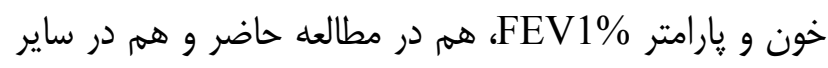
مطالعات در بيماران COPD ضعيف و يا بدون رابطه معنى دار

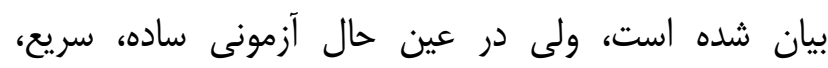

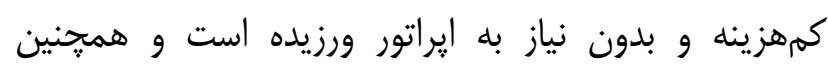

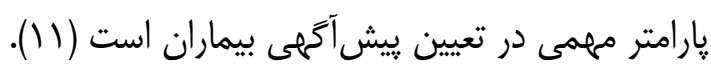

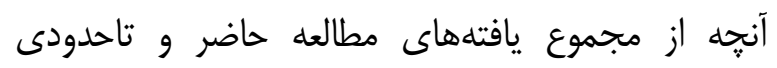

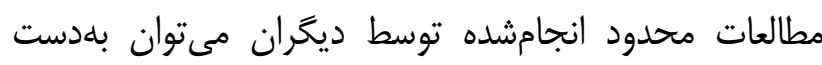

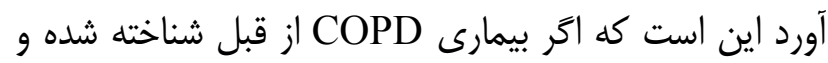
ميزان FEV1\% مشخص باشد، در بيخيرى بيماران مىتوان

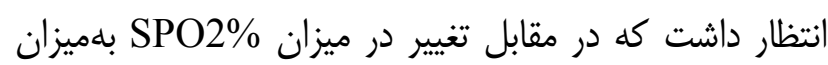

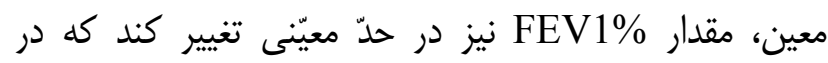

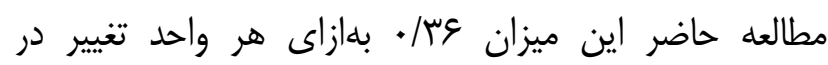

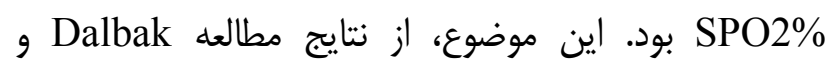

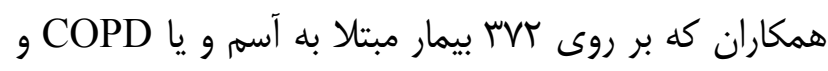

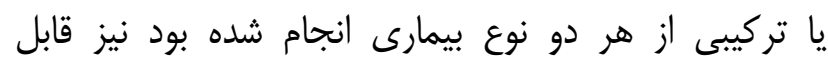

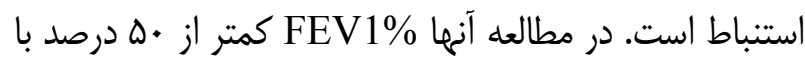
كمتر از ٪902\% در مطالعه حاضر عامل محدودكننده اصلى، عدم عدم امكان

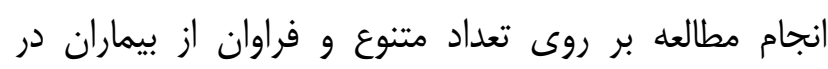

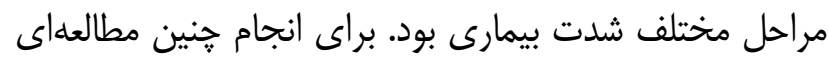

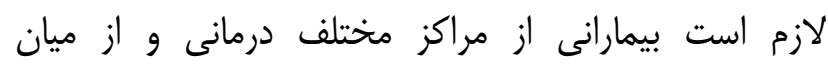
جمعيتهاى بزرى انتخاب شوند.

\section{نتيجه كيرى}

مقدار SPO2\% با مقدار FEV1\% رابطه ضعيف ولى مثبتى دارد و تا حدودى داراى قدرت ييشگَّيى براى تغييرات

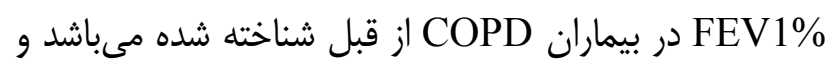

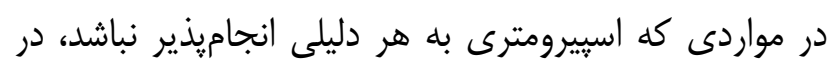

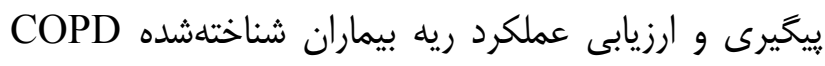
مىتواند مورد استفاده قرار كيرد.
با توجه به ميانخين FEV1\% بهدست أمده، بيماران مورد

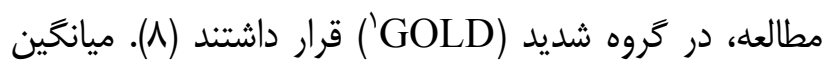

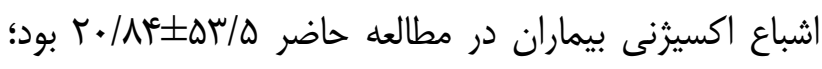

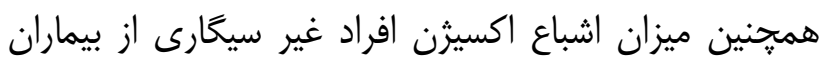

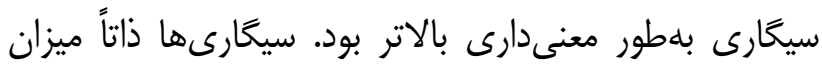

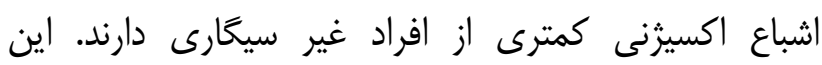

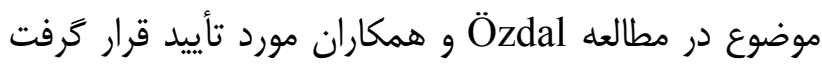
(9). با توجه به اينكه در بيماران مورد مطالعه، بين افراد سيكارى و غير سيگارى تفاوتى از نظر BMI) بيمارى، سن و همجنين VAS و ميزان FEV1\% مشاهده نشد، مىتوان تفاوتها در تغييرات اشباع اكسيزنى را مرتبط بان مين سيكار دانست.

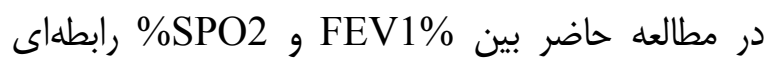
مثبت و ضعيف ولى معنىدار به دست آمد؛ همجنين نتايج

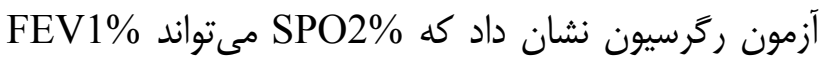
را بلهطور معنىدارى (البته در حد ضعيف) بيشبينى كندان COPD بعى از مطالعات نشان مىدهند كه در بيماران

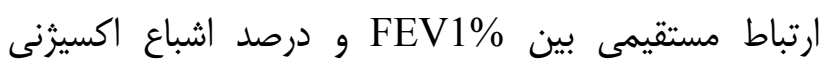
شريانى وجود دارد (·) (1). اين ارتباط در شرايط حملات تشديد COPD بيمارى COPD نسبت به شرايط عادى در بيماران

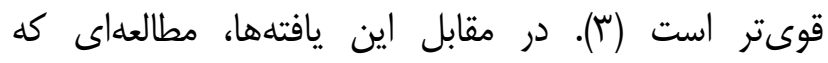
Garcia-Pachon קالساكسيمترى در بيماران COPD انجام دادند نشان داد،

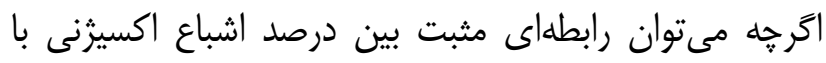
يالساكسيمترى و FEV1\% نشان داد، ولى اين ارتباط آنقدر از حساسيت و ويزگى برخودار نيست كه بتواند جايخزين اسييرومترى باشد و يا حتى بتوان بر مبناى آن بيماران نيازمند

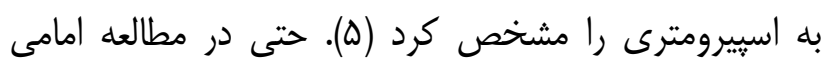
اردستانى و همكاران، هيج رابطه مثبتى بين FEV1\% و اشباع اكسيزنى در بيماران COPD غير بسترى مشاهده نشد ارند

\footnotetext{
${ }^{1}$ Global Initiative for Chronic Obstructive Lung Disease
} 
واحد توسعه تحقيقات بالينى بيمارستان وليعصر (عج) وابسته

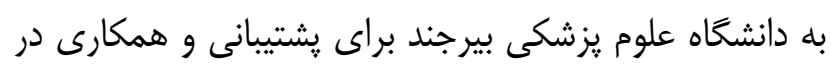
تقدير و تشكر طول دوره مطالعه اعلام مى دارند. نويسندكان مقاله، از معاونت يثروهشى دانشكاه علوم

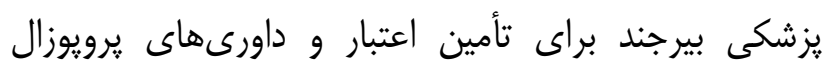

\section{تضاد منافع م:أع}

نويسندكان مقاله اعلام مى دارند كه هيج گَونه تضاد منافعى در

$$
\text { يثزوهش حاضر وجود ندارد. }
$$

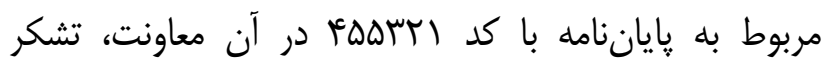

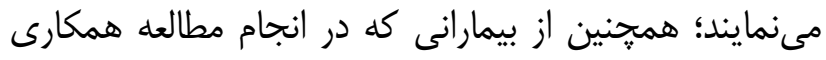

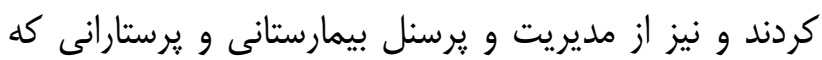

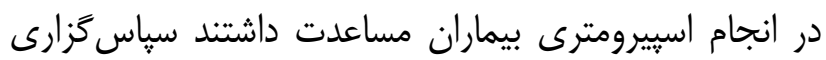

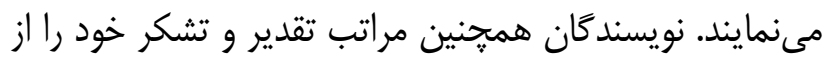

1- Miravitlles M, Vogelmeier C, Roche N, Halpin D, Cardoso J, Chuchalin AG, et al. A review of national guidelines for management of COPD in Europe. Eur Respir J. 2016; 47(2): 625-37. DOI: 10.1183/13993003.01170-2015.

2- Agustí A, Celli B. Natural history of COPD: gaps and opportunities. ERJ Open Res. 2017; 3(4). pii: 00117-2017. DOI: $10.1183 / 23120541.00117-2017$.

3- Schermer T, Leenders J, in 't Veen H, van den Bosch W, Wissink A, Smeele I, et al. Pulse oximetry in family practice: Indications and clinical observations in patients with COPD. Fam Pract. 2009; 26(6): 524-31. DOI: 10.1093/fampra/cmp063.

4- Kelly AM, McAlpine R, Kyle E. How accurate are pulse oximeters in patients with acute exacerbations of chronic obstructive airways disease? Respir Med. 2001; 95(5): 336-40. DOI: 10.1053/rmed.2001.1046

5- Garcia-Pachon E. Can pulse oximetry select patients for screening spirometry? Prim Care Respir J; 2004. 13(3): 1558 .

6- Ardestani ME, Abbaszadeh M. The association between forced expiratory volume in one second (FEV1) and pulse oximetric measurements of arterial oxygen saturation (SpO2) in the patients with COPD: A preliminary study. J Res Med Sci. 2014; 19(3): 257-61

7- Moreira MÂ, de Medeiros GA, Boeno FP, Sanches PRS, da Silva DP, Müller AF. Oxygen desaturation during the six-minute walk test in COPD patients. J Bras Pneumol. 2014; 40(3): 222-8. [English, Portuguese] DOI:10.1590/S180637132014000300004 .

8- Arkhipov V, Arkhipova D, Miravitlles M, Lazarev A, Stukalina E. Characteristics of COPD patients according to GOLD classification and clinical phenotypes in the Russian Federation: the SUPPORT trial. Int J Chron Obstruct Pulmon Dis. 2017; 12: 3255-3262. DOI:10.2147/COPD.S142997

9- Özdal M, Pancar Z, Çinar V, Bilgiç M. Effect of Smoking on Oxygen Saturation in Healthy Sedentary Men and Women. EC Pulmonol Respir Med. 2017; 4(6): 178-82.

10- Melbye H, Al-Ani S, Spigt M. Drop in lung function during asthma and COPD exacerbations - can it be assessed without spirometry?. Int J Chron Obstruct Pulmon Dis. 2016; 11: 3145-3152. DOI:10.2147/COPD.S123315

11- Ahmadi Z, Sundh J, Bornefalk-Hermansson A, Ekström M. Long-Term Oxygen Therapyvs 15 h/day and Mortality in Chronic Obstructive Pulmonary Disease. PLoS One. 2016; 11(9): e0163293. DOI: 10.1371/journal.pone.0163293.

12- Dalbak LG, Straand J, Melbye H. Should pulse oximetry be included in GPs' assessment of patients with obstructive lung disease? Scand J Prim Health Care. 2015; 33(4): 305-10. DOI:10.3109/02813432.2015.1117283. 